\title{
MYTHES, VARIANTES, INTERPRETATION OU DE LA CIRCULATION DU SENS
}

Frédéric François"

RÉSUMÉ: On tente d'illustrer l'impossibilité de donner du mythe une définition univoque en développant en particulier quelques oppositions comme mythes de l'autre / mythes de nous, mythes dominants et dominés, sociaux et individuels.

De toute façon les mythes ne peuvent que se transformer. Par exemple, dans l'apparition de mythes "scientifiques" à notre époque.

MOTS-CLÉS: mythe; récit; société; individu; sacré; profane.

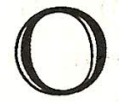

n est tout d'abord abattu par la multiplicité de ce qui peut s'appeler "mythe". On peut décider: "le vrai mythe c'est..., les autres ça n'en est pas". On peut au contraire penser qu'il y a des façons différentes d'être "mythe" comme d'être "objet de croyance" ou d'être "symbole" et que c'est cette relation d"air de famille", voire même de famille où on se bat et non de notion claire qui est constitutive de l'objet.

Je dis Dionysos, Edipe, Jésus-Christ, Don Juan, Barbe-bleue, la conquête de l'Ouest, Barbie 1 (le tortionnaire), Barbie 2 (la poupée), la societé sans classes, Julien Sorel, les trois petits cochons, le petit Chaperon rouge: tout cela est ou plutôt peut être considéré comme mythe, mais pas de la même façon. C'est en fonction de cette diversité qu'il me semble mal venu et en plus impossible de commencer par une définition. Plutôt, on voudrait tourner autour de quelques aspects dans la famille de sens du "mythe", sans que l'ordre des entrées soit justifiable.

1.) On commencera par distinguer "mythes de l'autre", "mythes de moi ou de nous". Le Rameau d'or de Frazer nous donne clairement des

(*) Faculté des Sciences Humaines et Sociales - Sorbonne V. 
FRANÇOIS, Frédéric. Mythes, variantes, interpretation ou de la circulation du sens.

mythes comme mythes des autres. Les Mythologies de Roland Barthes se situent dans notre société, mais sont quand même pour l'éssentiel "des autres". Il faut d'abord reconnaître qu'il y a forcément des mythes de nous, par opposition à l'idée simple et répandue: les mythes, les illusions sont de l'autre ou de nous comme autres, avant, quand on était enfant. Le Christ, Eedipe, Don Juan, l'apocalypse atomique, le "vilain figure du mal", j'ai nommé Barbie 1 sont "de nous", bien sûr à des titres divers.

2.) Comme figure du mal, je préfère Saddam Hussein, parce que ça nous rappelle que les mythes ne fonctionnent pas de la même façon pour tout le monde ni à tous les moments. Il y a une dizaine d'années Saddam Hussein était le rempart laïque de la civilisation occidentale contre l'Islam terroriste iranien. Il a été, il est encore l'incarnation de l'Islam bafoué et vengeur contre le démon occidental pour des millions de Mahométans. Ça nous rappelle aussi, qu'à l'intérieur d'une collectivité, un même mythe fonctionne différemment. Je pense que peu d'entre nous sommes des sectateurs de Saddam Hussein. Mais nous ne le démonisons pas tous, je suppose, de la même façon. Mythe d" ego" n'est pas la même chose que mythe de "nous". C'est aussi un bon exemple pour nous rappeler qu'il n'y a pas d'un cotê le réel, de l'autre le mythe. Certes, cette distinction est celle qu'on retrouve dans certains maniements du mot "mythe": autrefois, on croyait que ce qu'on trouve au début de Tite-Live, Romulus, et Rémus, les Horaces et les Curiaces faisaient partie de l'histoire romaine comme César ou Auguste. Maintenant, on pense que "ce n'était que des mythes". En même temps, qu'il s'agisse de vérité factuelle ou "mythique", il reste quelque chose de commun: un récit fondateur. On le sait: tous les Chrétiens n'ont pas le même type de croyance dans la vérité factuelle de la Bible. Ça ne les empêche pas de "croire". Ainsi, reconnaître que des mythes sont de nous 
Revista da ANPOLL, $\mathrm{n}^{\circ} 3$, p. 63-83, 1997

ne suffit pas, parce qu'ils ne le sont pas de la même façon, au même titre. D'où la nécessité de continuer à distinguer.

3.) D'abord, le mouvement de rejet des mythes fait partie intégrante de leur vie. Les mythes des autres conçus comme exemple de la bizarrerie humaine. En toute religion, il y a tendance à l'iconoclasme du mythe: comment les autres peuvent-ils croire des choses pareilles? Jésus-Christ fils de Dieu, Dieu lui-même est un scandale pour toute personne, juive ou musulmane qui prend un peu au sérieux la transcendance de Dieu: la raison dit qu'évidemment"ça n'est qu'un prophète. Ou encore les Romains adorateurs de Dieux au pluriel, objet d'exécration ou de ridicule pour les monothéistes, se moquaient de ce qu'on n'appelait pas encore les primitifs, mais les barbares qui, à travers leurs mythes adoraient le soleil ou une pierre. Imaginez un adolescent qui ne changerait absolument pas à l'égard de ses mythes d'enfance. Vous seriez inquiets. Même si j'ai de la peine à dire sur quel mode ça se passait, je peux me souvenir de mes mythes passés et les tenir à distance ou ne plus du tout comprendre quel a été leur pouvoir. Babar et puis aprés, moins avouable, Le Prince Eric, Le bracelet de vermeil, les récits de batailles navales des deux guerres mondiales. Mais aussi, des morceaux de mythes plus endogènes. Ou encore, c'est une évidence qu'on ne peut pas croire comme les premiers croyants. Lintégrisme, le refus de tout changement, qu'on rencontre sans doute en toute croyance instituée, est un rêve fou: la croyance bouge par le fait même qu'elle se transmet.

4.) Il faut donc distinguer mythes des autres, mythes de nous et mythes de moi. Mais surtout se demander selon quelles modalités les mythes sont "mythes de moi". Lacan parlait de "mythe individuel du névrosé", mais cela ne signifiait pas que ce mythe était purement de lui. D'une part, les éléments du mythe ne sont pas créés ex nihilo, mais d'autre part, qu'un mythe fasse ou non l'objet d'une reconnaissance institutionnelle, le fait 
FRANÇOIS, Frédéric. Mythes, variantes, interpretation ou de la circulation du sens.

qu'il soit pas qu'une "histoire que racontent les vieux" se passe bien en quelque façon en un "ego". On peut proposer que le statut de tout mythe qui fonctionne est d'une part de venir d'ailleurs, mais aussi d'être repris par un sujet particulier et de n'exister que dans ce mouvement. Ce qui n'empêche pas le "mythe individuel" de ne pas avoir forcément ni même le plus souvent la forme d'un mythe constitué, d'être plutôt un schème interprétatif. Quand le mythe individuel se constitue en récit structuré, affirmé publiquement, ça pose généralement des problèmes. Sauf si ça se passe dans un cadre spécialisé. Mais il me semble que, le plus souvent, nous avons la plus grande difficulté quand on nous pose la question à l'égard d'un mythe public dont nous participons "mais toi, tu y crois vraiment?" De toute façon, comme dans la "macro vie sociale", il y a la différence entre le mythe dominant, le mythe dominé, le mythe balbutiant, celui qu'on peut proclamer, celui qu'on cache. Ce qui fait que, par exemple, je ne transformerai pas la nature de cet exposé pour vous raconter le triste devenir des mes mythes. Une remarque générale plutôt. Personne ne coïncide complément avec soi-même et il est difficile ou impossible de dire quel est notre lien à notre propre croyance (est-ce le bon mot?) en ce qui concerne les mythes qui sont centraux dans notre perception même du "réel". En tout cas, cela se passe dans un espace discursif qui n'entre pas dans la dichotomie réel au sens de trivial quotidien / irréel, au delà, fictif. Comment dire exactement cet espace où le croyant retrouve dans le réel ce qu'on lui a raconté de la Providence divine ou bien où chacun de nous réalise ce qui lui a été annoncé par la saga familiale? Ou, au contraire, se construit comme le héros qui échappe à la malédiction annoncée.

Certes, il y a aussi la croyance qu'on oublie dans le cours ordinaire de la vie et qu'on ne se rappelle qu'à des moments précis. Je ne pense pas que la résurrection de la chair soit à chaque instant présente à l'esprit des chrétiens, ni que les anges ou le diable soient objet de croyance de la même façon que le Christ ou Dieu. La forme exacte de la trinité, c'est 
encore autre chose. Ceci pour dire que dans le livre, lieu de transmission, la croyance est unifiée; elle ne l'est pas pour autant en chaque croyant.

5.) Et il y a bien d"autres relations entre "mythe des autres"et "mythes de nous". Il y a aussi l'opposition entre le moment d'autrefois où le mythe était fort et maintenant où il est faible. Tous les discours où on évoque la "vraie foi" des autres.

Et il y a alors le discours de l'autre comme étant au contraire ce dont nous sommes devenus incapables. Une histoire hassidique, dont je ne sais pas très bien d'où elle me vient.

"Quand le grand-père du père de mon père avait quelque chose à faire, il allait dans la forêt, et il allumait un feu sous un arbre bien précis. Il se mettait en prière. Quand il avait fini, il savait ce qu'il allait faire.

Quand le père du père de mon père avait quelque chose à faire, il allait dans la forêt, il ne savait plus où était l'arbre. Il allumait un feu, se mettait en prière et quand il avait fini, il savait ce qu'il avait à faire.

Quand le père du mon père avait quelque chose à faire, il allait dans la forêt, se mettait en prière, et rentrait chez lui en sachant ce qu'il avait à faire.

Quand mon père avait quelque chose à faire, il n'allait pas dans la forêt, car celle-ci n'existait plus. Alors, il priait, et quand il avait fini, il savait ce qu'il avait à faire.

Quand à moi, il n'y a plus de forêt et je ne sais plus prier.

Mais je sais raconter l'histoire".

On retrouve ici le problème de la place particulière de celui qui raconte l'histoire: une histoire est une tradition, qui se modifie quand on la raconte. Et celui qui la raconte, ne peut pas, en même temps, ne pas se montrer comme digne ou non digne de la raconter:

6.) Poser les mythes comme à la fois "des individus" et "sociaux" est une chose. Mais peut-on préciser leur mode d'être social? J'ai pu lire 
FRANÇOIS, Frédéric. Mythes, variantes, interpretation ou de la circulation du sens.

dans un recueil de mythes grecs (Burn 1994: p.14) "On a récemment défini les mythes comme des 'récits traditionnels pertinents pour la societé"?" Il n'est cependant pas question d'être durkheimien, de poser une équivalence entre "le" mythe, "le" sacré et "la" société, ne serait-ce que parce que nous appartenons à plusieurs sociétés: la famille, la nation, les relations de travail, éventuellement une église, l'humanité et pas de la même façon. Reste qu'il est légitime de distinguer le mythe étayé par une pratique sociale et le mythe non-étayé par une pratique sociale. L'histoire de Don Quichotte est bien celle de l'homme dont le mythe fondateur ne coïncide pas avec les mythes environnants. Et, à l'intérieur même de la société, il y aura la distinction entre mythes facultatifs et nécessaires, imposés. Il y a une incertitude sur le mode d'être du mythe en moi, en particulier sur la modalité de ma croyance, mais sa façon d'être "dans" la société n'est pas non plus univoque.

7.) Pour aller d'un autre côté, il y a des mythes à forme narrative. Mais tout récit n'est pas mythe et tout mythe n'est pas récit.

On voit, en gros, de quoi il s'agit. Le mythe, c'est le récit comme fondateur ou plutôt comme sacré (on reviendra sur ce terme) par opposition à l'anecdote, à la distraction, à l'histoire drôle c'est moins sûr. (Car, justement, il se peut très bien qu'une "histoire drôle" fonctionne pour tel ou tel d'entre nous comme un "mythe": c'est-à-dire comme un texte qui manifeste sous cette forme ce qui ne pourrait pas être manifesté autrement, en particulier pas sous forme de discours conceptuel explicite. Ce n'est pas par hasard, selon la formule consacrée, que le Mot d'esprit et ses rapports avec l'inconscient de Freud est composé à plus de $80 \%$ d"'histoires juives").

Mais un tel discours fondateur n'est pas forcément un discours narratif. On peut néanmoins le considérer comme mythe. Dans sa fonction de fonder le réel, il s'étaye sur une autorité, constitue une obligation symbolique dans tel ou tel groupe tout autant qu'un mythe narratif. Toutes les formules du type "l'essence de l'homme c'est le travail, le désir, 
l'inconscient, l'appartenance à une communauté, le désir secret du divin qu'il ignore, la raison..." quand ils fondent une communauté peuvent être appelés mythes publics. Mais ils peuvent aussi constituer mon "mythe personnel". Dans un cas comme dans l'autre, ils ont leur talon d'Achille: ils sont soumis à la critique sur le mode du discours du "et alia": si on définit l'homme par le travail, par la lutte pour la reconnaissance ou par le manque de Dieu "et alors, moi, quand je joue, quand je dors, je ne suis plus un homme?". Le mythe n'est jamais le "tout du réel". Le "plus important" est-il dans les "grands mots" ou dans les "petits mots"?

A l'intérieur d'un certain désenchantement du monde et des formes narratives, de grandes figures organisatrices "laïques": la société sans classe, le libre-échange, l'humanité sans guerre, l'homme cartésien maître et possesseur de la nature ont ou ont eu ce statut "mythique" de discours qui compte, avec la relation compliquée qu'on a évoquée: bien sûr tout le réel n'est pas comme ça, mais on en perçoit déja des signes. Ces discours ne sont pas narratifs, mais ils ont relation au temps et à la réalité-irréalitésurréalité: ce n'est plus le passé qui importe, c'est l'avenir.

8.) La première caractéristique de l'analyse structurale des mythes a été d'être émerveillé par les récurrences. C'est assurément remarquable qu'on retrouve dans d'autres civilisations des thèmes mythiques semblables à ceux de la Bible et plus généralement qu'on les retrouve dans des civilisations différentes entre lesquelles il ne peut pas y avoir eu transmission. Jung par exemple a beaucoup insisté là-dessus. A sa suite Drewermann (1986) a été très frappé par la plus grande ressemblance du récit de la Vierge-mère et de la naissance de pharaon que de la continuité anciennouveau testament. Mais on peut aussi rappeler (Gustav 1941)) que Jupiter a été enfant et non seulement père barbu. Le thème du Dieu enfant est universel.

La plupart des mythologues présentent les mythes sous la forme d'une série de chapitres notionnels. Ainsi le livre de Mircea Eliade (1949) 
FRANÇOIS, Frédéric. Mythes, variantes, interpretation ou de la circulation du sens.

va traiter du "ciel, dieux ouraniens, rites et symboles célestes", "le soleil et les cultes solaires", "la lune et la mystique lunaire"..."la terre, la femme et la fécondité". Mais aucune mythologie n'est pure et simple cosmogonie. Il y a du cosmos. Il y a aussi des événements humains ou quasi-humains. Et le problème devient plus compliqué lorsqu'on se demande comment tout cela peut s'unifier. Drewermann écrit (op. cit. p.43) "Ces centres de gravité thématiques (sous-jacents aux récurrences mythiques) prédonnés de la vie humaine sont tout naturellement constitués des expériences les plus intenses, comme la naissance, la maturation, l'amour, la mort, le danger, le salut, la faute, la réconciliation etc..., et c'est surtout dans des événements biologiques, comme la génération, la conception, la maternité, l'enfance, la parenté et la descendance, que le mythe verra préfigurations symboliques du sens à donner au monde et de l'explication du monde". On peut à la suite d'Erwin Strauss rappeler: "c'est l'homme qui pense et non le cerveau". Il faudrait ajouter: c'est l'homme qui pense et non l'esprit. Si nous n'avions pas de mains, de pieds, de sexe, si nous n'étions pas des corps d'enfants ou de vieillards, nous n'aurions rien à penser.

On notera en même temps que dans cette recherche du "même" se perd le statut du récit mythique, qui fonctionne effectivement. Il y a aussi la différence entre noms communs qui sont "la lune" et "la femme" et les noms propres qui sont "Séléné" ou "Vénus". Noms propres qui ne le sont justement pas de la même façon que les noms de vous et de moi.

L'explication bute. Deux aspects peuvent peut-être ici être éclairants.

9.) D'abord, plutôt que d'opposer simplement la structure abstraite au récit concret, il me semble que dans le cas du mythe-narration, on peut distinguer une histoire ou un "paquet d'histoires" (plus noblement appelé "cycle"): en particulier, le grand personnage mythique (le Christ, Don Juan, (Edipe) ne se réduit pas à être le héros d'un récit, unique, assignable. Par exemple, dans les quatre évangiles il y en a un où il y a un bon et un mauvais larron (Luc, 23, 40) deux où il y a des larrons 
indifférenciés (Marc et Matthieu) et un, qui appartient souvent à un autre genre (celui de St. Jean) où il n'y a pas de larrons du tout. Ou bien, chez Luc et Mathieu le Christ crucifié appelle "Eli, Eli lamma Sabbachtani" (Seigneur, pourquoi m'as tu abandonné?), pas dans les deux autres évangiles. On le voit, la variante ne concerne pas ici des phénomènes secondaires.

Les mythologues ont en un sens à juste titre reproché à Freud d'avoir peu insisté sur le sort regrettable que les parents avertis par l'oracle avaient reservé d'abord à Eedipe ou d'avoir omis complétement le devenir d'Eedipe aveugle. En même temps, le reproche est absurde: au moment où on raconte, on ne peut raconter qu'une des versions, pas tout le cycle. Un peu comme on ne peut pas raconter en même temps toutes les fins du "Petit Chaperon rouge", mais, en même temps, ces autres versions sont à l'horizon du récit.

10.) Et puis, il y a une différence entre un récit, raconté hic et nunc, et une structure de récit. Evoquer "Don Juan", ce n'est pas la même chose que dire "séducteur". Et présenter telle "incarnation" de Don Juan pas la même chose qu'évoquer "Don Juan" en général. Ce qui nous conduit, me semble-t-il, à reconnaître qu'on ne peut séparer le récit de mythe de son style narratif.

Il me semble que cette dualité entre témathique et stylistique est souvent oubliée. D'abord, hors langage: le mythe n'est pas indifférent au medium. La Vierge peinte et le récit de l'annonce faite à Marie. Et puis, ensuite, telle annonce faite à Marie, qui pourra être pour nous la Vierge par excellence.

On lie souvent mythe et rite. Mais que serait la crucifixion racontée sans crucifix ou sans tableaux représentant la crucifixion? Les mythes s'incarnent dans des images ou des objets. Mais cela vaut aussi pour des mythes non reliés à un culte. Ils s'incarnent aussi: Don Juan opéra et Don 
FRANÇOIS, Frédéric. Mythes, variantes, interpretation ou de la circulation du sens.

Juan pièce de théatre ou "roman". De même que dans le cas de la pièce de théatre porté par tel ou tel acteur: Ou encore par un film. Sans cette relation à une forme particulière, le mythe est une représentation traductible, il. n'a pas d'effet. Ou un contre-effet. Je pense au problème de la présentation de la statue du commandeur. Quelles conditions pour que sa "grandeur mythique" ne vire pas au comique? Mais ça ne signifie pas seulement qu'il y a des formes qui font plus ou moins d'effet. Ça signifie aussi que la circulation est constitutive du mythe.

Un peu comme le va-et-vient entre l'effet du rệve et sa reprisemodification dans le récit de rêve. Ou la différence entre oral er écrit, qui recoupe en quelque sorte celle entre mythe privé et mythe public, au moins dans le cas de l'écrit lu solitairement, en même temps que le mythe écrit est du côté de l'unification alors que les variantes sont liées à la traduction orale. Et puis l'oral comme l'écrit associent au mythe une corporéité propre. La voix de la mère qui raconte à l'enfant qui s'endort, le corps de l'acteur tragique, mais aussi le livre sacré, en un sens unique. Un recueil de mythes n'est pas mythe en fonctionnement. Ou, si l'on veut, lire des mythes à la file leur retire l'essentiel de leur mode de fonctionnement. Comme lire un roman n'est pas la même chose que de lire un résumé de roman dans le LaffontBompiani pour faire une dissertation ou faire semblant de l'avoir lu.

Ce lien à un medium correspond aussi au mode de présence de l'être mythique. Ce n'est ni un individu, ni une abstraction, le personnage du mythe existe autrement. Pour utiliser un mot encore plus variable que "mythe", on dira que le mythe n'est pas signe, mais symbole.

\section{1.) Dans quelle mesure un mythe est-il un symbole?}

En entendant par là quelque chose qui transcende l'opposition vraifaux, l'opposition signifiant-signifié, l'opposition général-particulier, l'opposition de moi-de l'autre en ce qu'elles ont de trivial. On peut ici proposer qu'un mythe est une sous-espèce des signes denses, qui fonctionnent par leur matérialité même. Je prends des exemples dans la 
vie de tous les jours: une maison, un pont, un chemin dans la forêt au delà du bien connu et de l'utilitaire fonctionnent en même temps comme significations denses, par exemple comme lieu du repos, de la confiance, comme ce qui lie, comme là où on peut se perdre avec sécurité. On notera d'une part qu'une maison particulière, un chemin particulier ou un pont particulier soit de notre propre vie soit donnés par tel ou tel artiste sont des maisons, des ponts, des chemins par excellence et encore davantage ils manifestent l'accueil, le passage, le cheminement sans entrer dans la dichotomie particulier-général. Ils sont densément métaphoriques. Le langage ici retrouve une façon d'être des signes non-langagiers: l'opposition du familier et de l'étrange ne vient pas du langage et ne se réduit pas à ce qu'on en dit.

Même s'il y a quelque chose de spécifique au symbole en tant qu'il passe par le langage. Certes, un texte comme la Bible comporte des tas de genres de discoures différents, mais pour l'essentiel (je ne discute pas ici de sa lecture comme vérité factuelle), elle présente l'aventure du peuple juif déplacé chez les Egyptiens comme une symbolique de l'humanité qui n'est pas autochtone. Mais, en même temps, ce peuple juif reste "à distance". De même qu'il y a une imitation de Jésus-Christ comme un modèle mais un modèle inatteignable. Le symbole est du côté du "de te fabula narratur". C'est ton histoire. Mais ça ne signifie pas simplement "identification".

L'histoire que raconte le mythe a certes un sens pour moi, mais elle reste histoire locale, lointane. Le récit symbolique n'est pas une allégorie. Dans la mesure où le sens de l'allégorie se réduit à la traduction conceptuelle générique que je peux en donner. Alors que si je supprime la matérialité du récit, il n'y a plus de sens du tout. Ce qui vaut pour le mythe comme pour la pierre sacrée ou le tableau. Ce à quoi me fait penser une maison ou une statue sera dit, plus ou moins bien, non présenté sous forme de maison ou de statue. Si la nature symbolique du mythe est difficile à saisir; c'est peut-être que le dit a plus de modes d'être différents que le seul montré. 
FRANÇOIS, Frédéric. Mythes, variantes, interpretation ou de la circulation du sens.

On pourrait en tout cas dire que le monde profane est celui où les choses ne sont que ce qu'elles sont, la vie de Jésus celle d'un pauvre juif dans l'empire romain alors que le monde symbolique est celui où il y a du sacré. Mais on retombe alors sur la difficulté ou l'impossibilité de fixer les notions une fois por toutes.

12.) Le mythe et l'opposition sacré-profane.

Ici, le difficile est aussi, comme pour "symbole", de ne pas fabriquer de fausses essences. Il y a un lien du sacré et de la force. Mircea Eliade (op. cit.) parle de "kratophanies" apparition du fort, mais le sacré n'est pas forcément puissant, même si le lien existe. De même, il y a un lien du sacré et du mystérieux. Il n'est pas nécessaire. De même, le sacré est souvent lié à l'au-delà. Mais on peut représenter un sacré de la seule présence de l'objet, l'atmosphère de la communauté ou la solitude. On dira, en tout cas, que tout ce qu'on considére comme sens non lié à un rapport moyenfin peut être appelé sacré. En ce sens, raconter un mythe (même si je peux toujours raconter pour édifier, pour amuser ou pour faire le malin) c'est présenter un objet en tant qu'il ne se réduit pas à un but ou un calcul.

Une société laïque et où le rapport commerçant domine n'est pas une société sans sacré, mais avec un sacré autrement institutionnalisé que, par exemple, dans une société essentiellement agricole ou guerrière. Reste que, sans doute sans très bien savoir pourquoi, nous considérons qu'un bébé n'est pas une merchandise comme les autres. Ou que nous ressentons, pas seulement pensons, que la vie humaine, est "respectable", avec généralement de grosses différences selon qu'elle est celle de proches ou non, mais c'est une autre histoire.

13.) Peut-être plus qu'une analyse en termes de différence des mythes, devrait-on parler d'une différence dans la lecture des mythes.

On peut lire ou entendre un récit comme me concernant, comme concernant les autres. 
Le mythe peut me frapper pour des raisons opposées: par sa proximité ou par sa distance en particulier. La conquête de l'ouest pour l'américain actuel qui se représente comme un descendant des conquérants en question et pour l'enfant français qui regarde à la télé un film de cow-boys.Il y aura toujours forcément une distance entre celui qui raconte et celui qui entend, entre celui qui entend pour la première fois et celui qui est habitué.

Ce qui nous conduit au problème interne de chaque société, où il y a des sujets savants, transmetteurs ou fabricants de mythes pour les autres. Je ne pense pas que tout mythe soit l'exploitation de la naïveté des travailleurs par la caste-support du discours reconnu, elle-même appuyée sur la classe des puissants et la soutenant, mais il est vrai qu'il y a une fonction institutionnelle des mythes ou plutôt des castes qui vivent de la transmission de mythes. Et on retrouve ici le rôle de ceux qui disent ou fabriquent des mythes "ready made" pour enfants. On peut s'en moquer. On peut aussi se demander de quelle nature est le savoir de ceux qui savent que "ça va marcher". Le problème est compliqué: le séducteur est, le plus souvent, j'imagine, séduit. Celui qui produit un film de cow-boys ou de science-fiction?

J'imagine toujours un pape proclamant du haut du balcon du Vatican que tout ça n'est que des fariboles, c'est peu probable. Même chose pour les pédagogues, les psychanalystes ou qui vous voudrez.

14.) Les mythes sont sous-tendus par l'opposition exotérique / ésotérique et donc par leur relation aux autres discours. C'est ainsi que les mythes se diversifient aussi selon l'interprétation qu'ils reçoivent, leurs relations aux autres discours qui les entourent. Pour prendre un exemple évident, le récit de la Genèse ne peut plus fonctionner de la même façon quand il est confronté à des discours "scientifiques" sur les galaxies, l'évolution des espèces ou les gènes. Qu'est-ce qu'un mythe dans une civilisation où il n'y a pas d'autres discours dominants et dans un monde où il y a d'autres discours dominants? Il est comique de se représenter leș 
FRANÇOIS, Frédéric. Mythes, variantes, interpretation ou de la circulation du sens.

pauvres sauvages se disant: comme on ne connaît pas la vraie origine du monde, on va se raconter des histoires.

Ceci m'amène à parler encore plus au-delà de ma compétence. Une des profondes raisons pour lesquelles il n'y a pas de primitif ni de mentalité pré-logique, ni de logique de l'enfant, c'est que l'homme n'a jamais une raison mais plusieurs. La raison de celui qui fabrique quelque chose, qui cherche son chemin, la raison de celui qui classe, la raison de celui qui donne sens à sa vie. Il y a des cas où être rationnel, c'est ne s'occuper que de ce qu'on regarde, d'autres cas où il s'agit de faire des analogies, d'autres cas où il s'agit de replacer le réel par rapport à un possible ou un impossible.

La caractéristique du temps actuel, c'est que la dissociation des diverses raisons atteint un degré extrême. Une raison mathématique, ellemême dissociée en raison calculante et raison inventante, une raison technique, une raison universalisante-commerçante, une réthorique codée des medias et la mise en contemporanéité des figures de l'humanité qui jusqu'ici ont plutôt foncionnées séparément. D'où le fait qu'on peut sans difficulté devenir bouddhiste ou mahométan, ce qui est assez plaisant, en tout cas nouveau. Le capitalisme marchand s'accompagne aussi d'un libre marché des mythes.

Mais ce n'est là que l'aspect extérieur de la chose.

En tout cas il y a une parenté profonde de la raison (d'une certaine raison) et de l'imaginaire: je suis comme les autres / différent des autres, je peux avoir dans mon espace imaginaire des autres que moi. Cette présence de l'absent n'est pas que le fait du langage. Mais celui-ci est un multiplicateur d'imaginaire. Il utilise les mêmes formes de signification pour nous renvoyer à de l'expérience banale: "le café est trop chaud"; et pour nous présenter des objets inatteignables: "l'amour mène le monde" ou "Dieu est partout". Si l'on veut, il n'y a pas pour lui de limites du "présentable". 
Codicille: ce n'est pas par infantilisme que nous avons des mythes. Mythe de vrai adulte qui ne se raconterait pas d'histoires et taperait 8 heures par jour sur son ordinateur. Ou qu'est-ce qu'être dans le réel"?

On voit bien ce que c'est que la fascination par l'image. On voit bien ce que c'est que l'imaginaire refuge. Mais on ne peut pas imaginer un réel qui ne serait que réel, sans futur, passé, possible, impossible, à fuir, à chercher.

Rester enfant, être bisexuel, être éternel, être animal ou être vent. Chacun d'entre nous n'est que quand il est autre chose que coïncidence à soi. Mais on connaît tous la femme enfant, qui l'est un peu trop, celui qui est viril de père en part, celui qui cherche à être complètement, physiquement bisexuel.

Avoir un horizon d'altérité, c'est autre chose que se prendre pour un autre.

15.) En ce sens, comprendre un mythe, c'est s'en constituer un analogon personnel.

Je fais appel ici à une citation de Wittgenstein (1930), dans le court ouvrage qu'il a consacré au livre de Frazer, le rameau d'or, qui, on l'a dit, présentait les mythes des autres comme l'exemple même de ce qui n'est pas "de nous".

J'applique au mythe ce qu'il applique au symbole religieux "Un symbole religieux ne se fonde sur aucune opinion... Et c'est seulement à l'opinion que l'erreur correspond... Bruler en effigie. Embrasser l'image du bien-aimé. Cela ne repose naturellement pas sur la croyance qu'on produit un certain effet sur l'objet que l'image représente... Songeons qu'à la mort de Schubert son frère découpa en petits morceaux des partitions de Schubert et donna à ses éleves préférés ces fragments de quelques mesures. Cette façon d'agir comme marque de piété, nous est tout aussi compréhensible que l'autre, celle qui consisterait à conserver les partitions 
FRANÇOIS, Frédéric. Mythes, variantes, interpretation ou de la circulation du sens.

intactes, à l'abri de tous. Et si le frère de Schubert avait brulé les partitions, cela aussi serait compréhensible comme marque de piété". Il ne s'agit pas de nier que tel ou tel puisse avoir l'opinion que bruler en effigie agit réellement sur l'autre. Mais cette opinion importe moins que la possibilité de faire sens dans un amour ou une piété. Il me semble que c'est sela que Wittgenstein veut dire. Et aussi, qu'il est à la fois fondamental qu'il y ait plusieurs histoires, plusieurs façons pour le frère de Schubert d'honorer la mémoire de son frère. Mais qu’il ne peut pas le faire de toutes ses façons à la fois.

Après tout, les disciples de Freud (1 p.142) pour son cinquantième anniversaire "lui offrirent où il figurait sur une face son portrait et sur l'autre CEdipe et le Sphynx, avec un vers de l'CEdipe-roi de Sophocle "cet expert en énigmes fameuses, qui était devenu le premier des humains". Ce qui pose quelques problèmes d'interprétation. Les disciples songeaient-ils au sort funeste d'CEdipe ou à la fidélité de sa fille dans ses mallheurs? Sans doute pas, les limites de ce que le mythe "donne à penser" sont opaques.

\section{6.) Mythe: sens ou effet?}

Après tout, on aurait pu commencer par là: un mythe qui fonctionne n'est pas une représentation ou pas essentiellement une représentation. Le frère de Schubert ou les disciples de Freud n'ont pu utiliser ces signes que parce qu'ils étaient liés affectivement à leur "objet", émus par lui, par un lien qui se trouve ensuite modifié par la matérialité du symbole. On est lié affectivement à la joie de la naissance du Christ ou à la douleur de la Vierge à la mort de son fils, sans s'occuper d'abord du mode d'existence exact de ces objets. Même chose pour mes mythes personnels peu dicibles: ils constituent l'atmosphère affective de notre vie. Et il en est de même pour les mythes vus de lion, esthétisés, par exemple les histoires d'ogre que je raconte aux petits enfants: il y a une différence fondamentale entre une peur ludique, cathartisée et pas de peur du tout. Ce qu'on voit dans le triomphe de l'enfant que dit "c'est pas pour de vrai". 
17.) Mythe, "contradiction" et irréductibilité du mythe.

Une des caractéristiques des "personnages de la mythologie", c'est qu'ils entrent dans des récits différents, non combinables même dans la "même histoire", mais qu'il serait ridicule d'appeler "contradictoires". On le sait, une certaine forme de raison a été fondée sur la phobie de la contradiction. Il s'agit de poser des êtres qui ne soient que ce qu'on en dit et pas autre chose. Ce que font, au moins à un certain niveau, les mathématiques. Peut-être que l'idéalisme, c'est ça. Le plus mauvais Platon: la réalité d'ici bas est changeante, là-haut les chose sont définitivement ce qu'elles sont, les bienheureux définitivement heureux. On pourrait peutêtre sans les appeler bons ou mauvais distinguer des mythes transparents ou épais. Les mythes transparents seraient les mythes univoques: le méchant qui n'est que méchanceté, la femme qui n'est qu'amour. Le mythe épais serait celui qui ne peut être conceptualisé, mais seulement raconté parce qu'il est vraiment contradictoire. Est-ce que ça n'est pas par excellence le cas du Christ? Si son humanité est superficielle, si c'est un truc planifié par Dieu pour sauver les humains, mais moins vrai que sa divinité, on frise le comique.

C'est cette irréductibilité du narratif comme opaque qui me semble caractériser les "grands récits".

Ainsi Abel et Caïn.

1 Or Adam connut Eve sa femme et elle conçut en engendra Caïn, et elle dit: j'ai acquis un homme avec l'aide de l'Eternel.

2 Elle enfanta encore son frère Abel; et Abel fut berger, et Caïn fut laboureur.

3 Or, au bout de quelque temps, Caïn offrit de la terre une oblation à l'Eternel;

$4 \mathrm{Et}$ Abel lui offrit, lui aussi, des premiers-nés de son troupeau, et de leurs graisses. Et l'Eternel eut égard à Abel et à son oblation.

5 Mais il n'eut point d'egard à Caïn, ni à son oblation; et Caïn fut fort irrité et son visage fut abattu. 
FRANÇOIS, Frédéric. Mythes, variantes, interpretation ou de la circulation du sens.

6 Et l'Eternel dit à Caïn: pourquoi es-tu irrité et pourquoi ton visage est-il abattu?

$7 \mathrm{Si}$ tu fais bien, ne reléveras-tu pas ton visage? Mais si tu ne fais pas bien, le péché est couché à la porte, et son désir est tourné vers toi; mais toi, tu dois dominer sur lui.

8 Et Caïn parla à Abel, son frère. Et, comme ils étaient aux champs, Cain s'éleva contre son frère Abel et le tua.

9 Et l'Eternel dit à Caïn: Où est Abel, ton frère? Et il répondit: Je ne sais. Suis-je le gardien de mon frère, moi?

10 Et l'Eternel dit: Qu'as-tu fait? La voix du sang de ton frère crie de la terre jusqu'à moi.

11 Et maintenant tu seras maudit de la terre, qui a ouvert sa bouche pour recevoir de ta main le sang de ton frère.

12 Quand tu cultiveras la terre, elle ne te rendra plus son fruit; tu seras vagabond et fugitif sur la terre.

13 Et Caïn dit à l'Eternel: ma peine est trop grande pour être supportée.

14 Voici. Tu m'as chassé aujourd'hui de cette terre, et je serai caché de devant ta face, et je serai vagabond et fugitif sur la terre; et il arrivera que quiconque me trouvera me tuera.

15 Et l'Eternel lui dit: C'est pourquoi si quelqu'un tue Caïn, Caïn sera vengé sept fois. Et l'Eternel mit à Caïn un signe, afin que quiconque le trouverait ne le tuât point.

16 Alors Caïn sortit de devant l'Eternel, et il habita au pays de Nod à l'orient d'Eden.

17 Puis Caïn connut sa femme, qui conçut et enfanta Henoc; or, il construisit une ville qu'il appela Henoc, du nom de son fils.

J'arrête, mais le récit ne s'arrête pas, avec la génération de Caïn. Chacun peut le percevoir à son mode. Ça ne ressemble pas à une structure narrative typique. Si c'est un "vrai" récit, c'est aussi, d'abord que ça comporte de l'opaque. Qu'on (je en tout cas) ne peut pas "donner une figure notionnelle simple" des rapports de l'Eternel et de Caïn. 
Lincompréhensibilité (partielle) est constituve du mythe par opposition à l'allégorie.

Dans ce récit, il y a de l'intelligible. Mais qu'en est-il de ce Dieu qui privilégie l'un des deux frères et son offrande? Qu'en est-il de la protection accordée à l'assasin? De ce signe qui le protège? De cette histoire violente qui se transforme en généalogie?

Face au mythe, on ne peut que supporter l'indétermination. Le petit enfant qui ne sait s'il faut rire ou pleurer. Renan dans la Préface desDialogues (Renan 1984) s'assimile à un bon curé de village qui ayant raconté la Passion à ses paroissiens leur dit "mes enfants, ne pleurez pas tant que cela, il y a bien longtemps que cela est arrivé, et puis ce n'est peut-être pas bien vrai".

Ça n'est pas au moment du mythe qu'on erre, mais au moment de la prédication, du sérieux. D'où le sentiment de gêne lorsqu'un auteur aussi sérieux que Freud nous raconte comme si on y était la horde primitive et le vilain père qui se garde toutes les femmes pour lui. Il me semble que dès qu'il y a conscience du temps on est dans la culpabilité et c'est très bien comme ça. On n'a pas besoin d'un meurtre primitif pour expliquer la culpabilité. De même qu'un mythe n'est pas vrai ou faux en lui-même. On n'a pas à se poser la question de savoir si "la femme"est vraiment tentatrice et si ce benêt d'Adam serait resté tout seul au Paradis terrestre (hypothèse tout aussi bizarre que celle de savoir ce qui serait passé si Judas n'avait pas trahi). Ce qui importe, c'est que la femme est ou peut être aussi représentée par un autre mythe, par exemple, celui de la Vierge, ou de Marie-Madeleine ou de Jeanne d'Arc. Et puis il y a Salomé... L'intérêt des mythes, c'est qu'il y en a toujours d'autres.

18.) Il me semble que si modernité il y a, son sort est en partie liée à un mode particulier d'existence des mythes qui serait celui de la littérature. Les raisons de l'opposition de Platon aux poètes et à leurs charmes sont compliquées. Reste que ce fabricant de mythes par excellence est aussi à 
FRANÇOIS, Frédéric. Mythes, variantes, interpretation ou de la circulation du sens.

l'origine de ce qu'on pourrait appeler la "haîne de la littérature". De même, on sait la longue hostilité de l'Eglise catholique aux comédiens. Ce ne peut être simplement au nom de la notion de divertissement. Il me semble qu'on peut essayer de penser l'Eglise catholique d'autrefois en relation à l'Islam actuel et à sa façon d'admettre la science comme technologie et à se heurter à la littérature comme forme fondamentale du pluralisme. Les religions du livre sont religions du livre unique (avec, il est vrai, beaucoup de commentaires). On ne peut pas imaginer une littérature du livre unique. Mais la littérature s'oppose aux religions monothéistes dominantes sur un autre point. Mircea Eliade écrivait (p.40) "En effet, l'une des principales différences qui sépare l'homme des cultures archaïques de l'homme moderne réside justement dans l'incapacité où est ce dernier de vivre la vie organique (en premier lieu la vie érotique et la nutrition) comme un sacrement".

Or; il me semble que, dans la mesure où la "littérature" a partie liée avec "le sacré", c'est dans le retour à un sacré immanent, face à ce que Nietsche appelait les arrière-mondes. Sacré immanent qui n'est pas forcément lié à la puissance, ni au durable. On peut ici se demander si on doit décrire notre époque comme l'époque de la disparition du sacré ou comme la multiplication des sacrés terrestres, sacré du banal et non de l'extraordinaire, du faible, du labile et non pas du fort, de ce qui apparaît et non de ce qui est caché, de ce qui est pluriel et non pas unique, ce qui donne à mieux entendre ce qui est toujours là et non révélation d'un "autre", en évitant tout prophétisme ou toute façon de présenter "le savoir" sousjacent réservé à une élite.

Mais plus fondamentalement, il me semble que l'approche littéraire des mythes a partie liée à l'individualisme. Non l'individualisme dont il est facile de se gausser, celui de l'individu qui ne s'occupe que de son intérêt, mais l'individualisme de celui qui sait que c'est dans sa lecture que ce qu'il lit fait sens, même si, naturellement, un homme sans tradition ou plutôt sans traditions au pluriel, ça n'existe pas. 
Le dernier point serait peut-être que c'est à travers la littérature et plus particulièrement le roman que nous nous lisons nous-mêmes dans notre pluralité, en particulier de sujet soumis aux modes technicocommerciaux de circulation des livres et de ses bien culturels, mais, j'espére, pas complètement.

Ce qui est sûr, c'est que tout discours, quand il ne l'est pas dès le début, peut se transformer en répétition, en ennui, en langue de bois, d'où l'urgence de se taire.

RESUINO: Tentamos ilustrar a impossibilidade de dar do mito uma única definição, desenvolvendo, especificamente, algumas oposicöes como mitos do outro / milos de nós, mitos dominantes e dominados, sociais e individuais.

De todo mado, os mitos não podem senão se transformar. Por exemplo, no aparecimento de mitos "científicos" em nossa época.

PALAVRAS-CHAVE: mito; narrativa; sociedade; indivíduo; sagrado; profano.

\section{BIBLIOGRAFIA}

BURN, L. (1990). Mythes grecs, tr:fi: Seuil 1994.

FREUD, S. Le mot d'esprit et ses rapports avec l'inconscient.

DREIVERMANN, E. (1986). De la naissance des dieux à la naissance du Christ, tr.fr. Seuil, 1992.

JUNG, C.-G. et KÉRÉNYI, C. (1941). L'essence de la mythologie, tr.fr. Payot, 1951.

ELIADE, M. (1949). Traité d'histoire des religions, Payot.

WTTTGENSTEIN, L. (1930). Remarques sur le rameau d'or de Frazer, tr.fr. L'âge d'homme, 1982.

RENAN, E. (1984). "Dialogues et fragments philosophiques", édition consultée: Histoire et parole, auvres diverses, introduction de Laudyce Retat, p. 659, Robert Laffont. 